\title{
Effective lifetime of minority carriers in black silicon nano-textured by cones and pyramids
}

\author{
V.F. Onyshchenko ${ }^{1}$, L.A. Karachevtseva ${ }^{1}$, O.O. Lytvynenko ${ }^{1}$, M.M. Plakhotnyuk ${ }^{2}$, O.J. Stronska ${ }^{1}$ \\ ${ }^{I} V$. Lashkaryov Institute of Semiconductor Physics, NAS of Ukraine, \\ Photonic Semiconductor Structure Department, \\ 41, prospect Nauky, 03680 Kyiv, Ukraine, \\ E-mail:lakar@isp.kiev.ua \\ ${ }^{2}$ Technical University of Denmark, \\ Department of Micro- and Nanotechnology, \\ Ørsteds Plads building 345 East, DK-2800 Kgs. Lyngby, Denmark
}

\begin{abstract}
We calculated the dependence of effective lifetime of minority carriers in black silicon nano-textured by cones and pyramids on the diameter of the cone base, the side of the pyramid base, the height of cone and pyramid. The numerical calculation shows that $n$-type polished plate of single crystal silicon and $n$-type plate of black silicon have a high minority carrier lifetime both in the bulk and on the silicon surface, indicating a high purity of both the bulk of silicon and its surface. However, the measured experimentally effective lifetime of minority carriers in the $n$-type black silicon is $1.55 \mathrm{~ms}$ and is determined by the surface lifetime. The measured effective lifetime of minority charge carriers in the $p$-type polished silicon is $1.24 \mathrm{~ms}$. The minority carrier lifetime in the bulk of the polished $p$-type silicon is lower than the surface one.
\end{abstract}

Keywords: effective minority carrier lifetime, excess minority carrier, black silicon.

Manuscript received 07.07.17; revised version received 01.08.17; accepted for publication 06.09.17; published online 09.10.17.

\section{Introduction}

Black silicon is a structured surface of silicon, which seems to be black due to the effective reduction of reflection [1]. The surface of black silicon is structured by cones, pyramids and pores [2-4]. The structured surface of silicon not only reduces the reflection of light but also scatters the latter, which increases the optical path of light and its absorption [2]. If we structure two silicon surfaces then we obtain a multiple reflection of light between these surfaces due to the fact that a part of the rays scattered by one surface falls onto another surface at angles greater than the critical angle of the total internal reflection and will be completely reflected [5]. Analytical calculations of the angular distribution of light in the volume of silicon structured by cones show that $70 \%$ of light can be subjected to internal reflection [5]. The reflection coefficient, transmittance and absorption of black silicon structured by cones, as well as the angular distribution of light in the silicon volume, can be calculated using the rigorous coupled wave analysis [2]. A black structured silicon surface that absorbs light in a wide range of wavelengths has found application in solar cells [5-7], photodetectors and image sensors with increased sensitivity [8-10]. Black silicon has a large surface area but due to qualitative passivation, the minority carrier lifetimes in solar cells with passivated black structured silicon surfaces are equal to milliseconds [11-13]. Having unique surface properties, black silicon quite unexpectedly found application in surface enhanced Raman spectroscopy [14] and terahertz emitters [15]. 


\section{The model of excess minority carrier distribution and effective lifetime of minority carriers in black silicon}

The effective lifetime of minority carriers in black silicon was calculated solving the diffusion equation for minority carriers using the models and methods to calculate the lifetime and minority carrier distribution in macroporous silicon and single crystalline silicon [16, 17]. The respective system of equations includes the diffusion equation for minority carriers in single crystal silicon and boundary conditions. Black silicon surface consists of cones and pyramids, which we separated into elementary cylinders. We analyzed elementary cylinders as an effective medium and sewed its bases. The stationary diffusion equation for minority carriers in elementary cylinder can be written as:

$D_{p} \nabla^{2} \delta p(x)-\frac{\delta p(x)}{\tau_{e f f, u c}}+g_{0 p} e^{(-\alpha x)}=0$,

where $D_{p}$ is the hole diffusion coefficient, $\delta p(x)$ - extra hole concentration, $\alpha-$ coefficient of silicon light absorption, $g_{0 p}$ - bulk generation of excess holes, $\tau_{\text {eff }, u c}-$ the effective minority carrier lifetime in elementary cylinder defined by the equation (12) according to $[16,17]$. The stationary equation of minority carrier diffusion in single crystalline silicon is similar to equation (1) but needs to put the volume lifetime instead the effective one. Boundary conditions are determined taking into account the balance of the excess carriers on the surface:

$g_{s}=e^{-1} j_{p}\left(x_{i}\right)+s_{p} \delta p\left(x_{i}\right)$,

where $e$ is the electron charge, $g_{s}$ - speed of the surface generation of the excess charge carriers, $\delta p$ - density of the hole current at the surface, $s_{p}-$ surface recombination velocity for holes, $\delta p\left(x_{i}\right)-$ concentration of excess holes on the surface, $x_{i}$ - coordinate of the selected surface.

The minority carrier diffusion in the cylindrical coordinates is

$D_{p}\left[\frac{\partial^{2} \delta p}{\partial r^{2}}(r)+\frac{1}{r} \frac{\partial \delta p}{\partial r}(r)\right]-\frac{\delta p(r)}{\tau_{b}}+g_{p}=0$.

Here, $g_{p}$ is the rate of spatially homogeneous generation of excess minority carriers, $\delta p(r)$ distribution function of steady-state excess minority carrier concentration as a function of the radius vector $r$. The general solution of the diffusion Eq. (3) is

$\delta p(r)=g_{p} \tau_{b}\left(1+C_{1} I_{0}(R)-C_{2} K_{0}(R)\right)$, where $R=r / L_{p}, L_{p}=\left(D_{p} \tau_{b}\right)^{1 / 2}$ is the minority carrier diffusion length, $C_{1}$ and $C_{2}$ are constants, $I_{\alpha}(R)$ and $K_{\alpha}(R)$ are the modified Bessel functions, $\alpha$ is the order of the Bessel function. If $R=0$, then $C_{2}=0$.

The boundary condition on the surface of a cylinder is

$D_{p} \frac{d \delta p}{d r}\left(r_{0}\right)=-s_{p o r} \delta p\left(r_{0}\right)$

where $r_{0}$ is the radius of a cylinder, $s_{p o r}$ - surface recombination velocity of minority carriers on the surface of a cylinder. From Eqs (4) and (5), it follows that

$$
C_{1} I_{1}\left(R_{0}\right)=-S_{p o r}\left(1+C_{1} I_{0}\left(R_{0}\right)\right),
$$

where $R_{0}=r_{0} / L_{p}$ and $S_{p o r}=s_{p o r} L_{p} / D_{p}$ are dimensionless quantities. The constants $C_{1}$ may be determined from Eq. (6). The solution of Eq. (3) is the distribution function of steady-state excess minority carrier concentrations as a function of the radius vector $r$

$(\delta p)_{S}(r)=g_{p} \tau_{b}\left[1-\frac{S_{p o r} I_{0}(R)}{I_{1}\left(R_{0}\right)+S_{p o r} I_{0}\left(R_{0}\right)}\right]$.

The average concentration of steady-state excess minority carriers in the cylindrical unit cell is

$\overline{(\delta p)_{S}}=\frac{g_{0 p}}{\tau_{e f f, u c}}=\frac{2}{r_{0}^{2}} \int_{0}^{r_{0}}(\delta p)_{S}(r) r d r$,

where $\tau_{\text {eff }}$ is the effective minority carrier lifetime. Using Eqs (7) and (8) as well as $\int_{0}^{r_{0}} I_{0}(R) r d r=\left.L_{b} r I_{1}(R)\right|_{0} ^{r_{0}}$, one can find the average concentration of steady-state excess minority carriers in the cylindrical unit cell:

$$
\overline{(\delta p)_{S}}=g_{p} \tau_{b}\left[1-\frac{2 S_{p o r} I_{1}\left(R_{0}\right)}{\left(I_{1}\left(R_{0}\right)+S_{p o r} I_{0}\left(R_{0}\right)\right) R_{0}}\right] .
$$

On condition that $R_{0}<0.1$, we replaced the cylindrical modified Bessel functions by the functions that can be written as $I_{0}(R) \approx 1, I_{1}(R) \approx R / 2$. Then, it is possible to present Eqs (7) and (9) as

$(\delta p)_{S}(r)=\overline{(\delta p)_{S}}=g_{p} \tau_{b}\left(1-\frac{S_{p o r}}{2^{-1} R_{0}+S_{p o r}}\right)$, 


$$
\begin{aligned}
& \frac{g}{(\delta p)_{S}}=\frac{g}{(\delta p)_{S}(r)}=\frac{1}{\tau_{e f f, u c}}=\frac{1}{\tau_{b}}\left(1+\frac{2 S_{p o r}}{R_{0}}\right)= \\
& =\frac{1}{\tau_{b}}+\frac{2 s_{p o r} L_{p}}{D_{p} \tau_{b}} \frac{L_{p}}{r_{0}}
\end{aligned}
$$

or

$$
\frac{1}{\tau_{e f f, u c}}=\frac{1}{\tau_{b}}+\frac{2 s_{p o r}}{R_{0}} .
$$

Let us obtain a simple expression to determine the effective minority carrier lifetime in a cylindrical unit cell.

The effective lifetime of minority carriers in black silicon was obtained as follows:

$$
\tau_{\text {eff }}=\frac{1}{h g_{0 p}} \int_{r_{0}}^{h} \delta p(x) d x,
$$

where $h$ is the sample thickness.

\section{The results of calculation of effective lifetime of minority carriers in black silicon}

For the numerical calculations, presented in Table, we used the data from the paper [18], where the thickness of the polished single crystal wafer and black silicon plate was $470 \mu \mathrm{m}$ for $n$-type and $p$-type silicon, and the concentration of excess carriers was $5 \cdot 10^{15} \mathrm{~cm}^{-3}$.

The effective lifetime of minority carriers for $n$ type polished plate of single crystal is equal to $5.64 \mathrm{~ms}$ and for black silicon wafer $-1.55 \mathrm{~ms}$, in polished plate for single $p$-type crystal $-1.24 \mathrm{~ms}$ and for black silicon wafer $-0.75 \mathrm{~ms}$, see Table.

Surface recombination velocity was calculated in [18] as $s_{\text {eff }}=h /\left(2 \tau_{\text {eff }}\right)$. The recombination velocity $s_{\text {eff }}$ was equal to $4.17 \mathrm{~cm} / \mathrm{s}$ for $n$-type plate of black silicon and to $15.16 \mathrm{~cm} / \mathrm{s}$ for $p$-type plate [18] (see Table).

Let's estimate relation between the concentration of recombining carriers on the side of the black silicon and the polished side using the formula $s_{2} / s_{1}=2 s_{\text {eff }}(\mathrm{Bl}) / s_{1}-1$, where $s_{\text {eff }}=h /\left(2 \tau_{\text {eff }}\right), \tau_{\text {eff }}=\tau_{s}=h /\left(2 s_{\text {eff }}\right), 2 s_{\text {eff }}=s_{1}+s_{2}$. The value $s_{1}$ is the surface recombination velocity on the polished plate and the polished side of black silicon. The value $s_{2}$ is the marked surface recombination velocity on the black silicon side of the plate and $s_{\text {eff }}(\mathrm{Bl})$ is the effective surface recombination velocity on the black silicon at all. The ratio $s_{2} / s_{1}$ is higher than 6 for a plate of black $n$-type silicon, whereas for $p$-type this ratio is 2.3 , according to Table.

Using numerical methods, models and techniques presented in $[16,17]$ we picked the volume lifetime $\tau_{b}$ and the surface recombination velocity $s$, so as to match the effective lifetime $\tau_{\text {eff }}$ of a polished silicon wafer and black silicon. For black silicon, the distance between cylinder centers is $0.13 \mu \mathrm{m}$, the cylinder diameter is $0.11 \mu \mathrm{m}$, the cylinder height is $0.5 \mathrm{~mm}$ for black silicon, according to [18]. We also considered black silicon square pyramids at the base instead cylinders. The pyramid height is equal to the cylinder height, the distance between pyramid centers is equal to the distance between cylinder centers. The base side of the pyramids is equal to the cylinder diameter. The bulk lifetime for monocrystalline silicon wafer and black silicon were the same. We obtained the minority carrier lifetime $0.03 \mathrm{~ms}$ for $n$-type silicon and $1.75 \mathrm{~ms}$ for $p$-type silicon (the concentration of excess carriers $5 \cdot 10^{15} \mathrm{~cm}^{-3}$ ) calculated using the numerical methods and models presented in $[16,17]$. The estimated rate of surface recombination on the material surface was $2.67 \mathrm{~cm} / \mathrm{s}$ for the $n$-type silicon and $4.35 \mathrm{~cm} / \mathrm{s}$ - for the $p$-type one, taking into account the same rate of surface recombination for single crystal wafer and for black silicon (Table).

Let's estimate relation between concentrations of recombining carriers on the black silicon side and on the polished side of silicon by using the formula specified above. The surface recombination velocity on the black silicon $n$-type surface plate is 7.14 times higher than that on polished surface (see Table). This result does not differ much from the calculations with effective values neglecting the volume lifetime. At the $n$-type black plate of silicon, the lifetime is high $(30 \mathrm{~ms})$, and therefore it does not affect the effective lifetime of minority carriers. At the same time, the surface recombination velocity for the $p$-type black silicon plate is 9.62 times higher than that for polished one and higher $s_{2} / s_{1}=2.31$ calculated for the effective values. In the latter case, the volume lifetime plays an essential role in the charge carrier recombination. Table also contains the lifetime of minority carriers at the surface, which shows the impact of the entire material surface on the recombination processes.

Table: Characteristics of polished and black silicon plates.

\begin{tabular}{|l|c|c|c|c|c|c|c|}
\hline & $\tau_{s}=\tau_{e f f}, \mathrm{~ms}[3]$ & $s_{e f f}, \mathrm{~cm} / \mathrm{s}[3]$ & $s_{2} / s_{1}$ & $\tau_{b}{ }^{*}, \mathrm{~ms}$ & $\tau_{s}{ }^{*}, \mathrm{~ms}$ & $s^{*}, \mathrm{~cm} / \mathrm{s}$ & $s_{2}{ }^{*} / s_{1}{ }^{*}$ \\
\hline$n-\mathrm{Si}$ & 5.64 & 4.17 & 1 & 30 & 6.95 & 2.67 & 1 \\
\hline $\mathrm{Bl} n-\mathrm{Si}$ & 1.55 & 15.16 & 6.28 & 30 & 1.63 & 2.67 & 7.14 \\
\hline$p-\mathrm{Si}$ & 1.24 & 19 & 1 & 1.75 & 4,26 & 4.35 & 1 \\
\hline $\mathrm{Bl} p-\mathrm{Si}$ & 0.75 & 31.3 & 2.31 & 1.75 & 1.32 & 4.35 & 9.65 \\
\hline
\end{tabular}

The values in Table were obtained by numerical calculations with account of the bulk lifetime and marked with the sign *. 


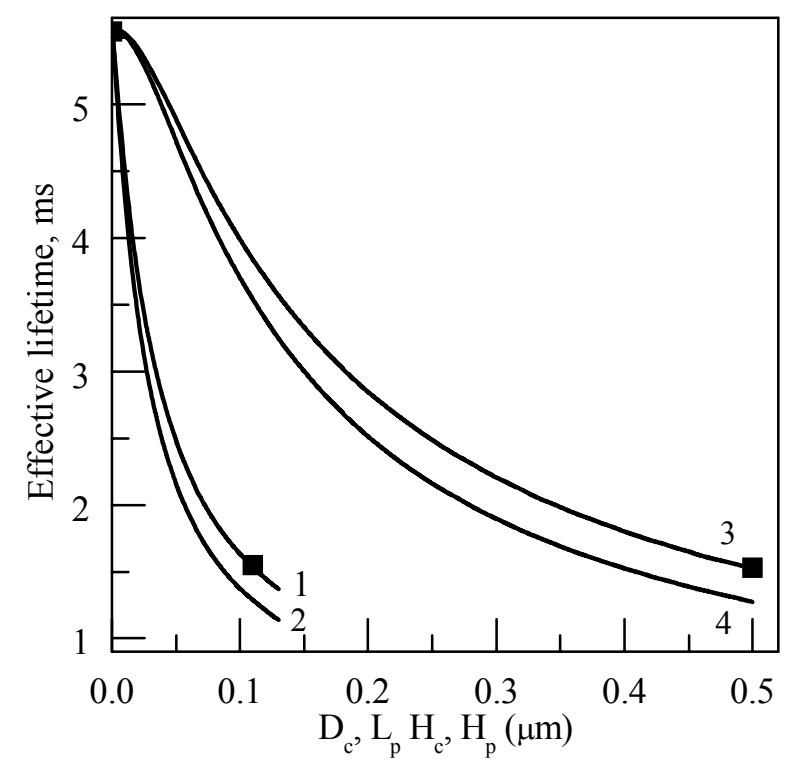

a)

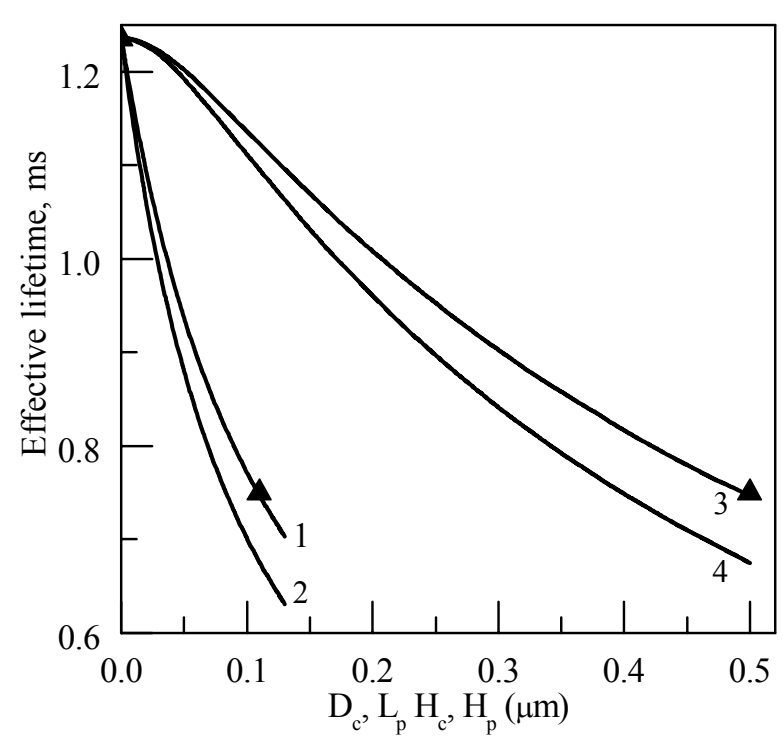

b)

The dependence of the effective lifetime of minority carriers from the base of the cone diameter circle (1), the base parties of the pyramid (2), the height of the cone (3) and pyramid (4) for $n$-type (a) and $p$-type (b) black silicon; signs - experimental data from [18].

Let's compare the volume lifetime $\tau_{b}$ and the minority carrier lifetime $\tau_{s}$ on the surface knowing that the effective lifetime $\tau_{\text {eff }}$ is less than the least one. The volume lifetime $\tau_{b}$ and the minority carrier lifetime $\tau_{s}$ are high in polished $n$-type monocrystalline silicon indicating both volume and surface material purity. But Table data show that the effective lifetime $\tau_{\text {eff }}$ is a little less than $\tau_{s}$. In the $n$-type black silicon plate, the effective lifetime $\tau_{\text {eff }}$ and the surface lifetime $\tau_{s}$ are almost identical. So, very pure material volume does not influence on recombination processes. The minority carrier lifetime in the polished $p$-type bulk silicon is lower than the surface lifetime $\tau_{s}$, which indicates that the concentration of surface recombination centers is less than the volume one. The minority carrier lifetime in $p$-type black silicon is lower than the volume lifetime $\tau_{b}$.

We calculated the dependence of the effective lifetime of minority carriers on the diameter of the cone base $D_{c}$, the base of the pyramid sides $L_{p}$ on the height of cones $H_{c}$ and pyramids $H_{p}$. We used the Table data and methodology for calculating the effective lifetime of macroporous silicon from $[16,17]$.

Figure shows dependences of the effective lifetime of minority carriers for $n$-type $(a)$ and $p$-type $(b)$ of black silicon on: the diameter of the cone base $D_{c}$ (curve 1 ), the base of the pyramid sides $L_{p}$ (curve 2), the height of cones $H_{c}$ (curve 3 ) and pyramids $H_{p}$ (curve 4 ). We have an effective minority carrier lifetime for polished monocrystalline silicon wafer at zero point. We obtained the effective minority carrier lifetime (Figure, signs) for black silicon (see [18] and Table) for the diameter of the cone base $D_{c} 0.11 \mu \mathrm{m}$ (curve 1 ) and for the height of the cone $0.5 \mu \mathrm{m}$ (curve 3).
Figure shows that the effective minority carrier lifetime in the black silicon wafer with cones (curves 1 and 3) is higher than the effective minority carrier lifetime on the surface with pyramids (curves 2 and 4). The effective photocarrier lifetime in the black silicon wafer sharply decreases (curves 1,2) with increasing the diameter of the circle base of the cone, the sides and base of the pyramid height difference between life times in a plate of black silicon cones and pyramids grows (curves 1,2 and 3,4).

The effective lifetime of minority carriers in the silicon wafer heights curves sharply decreases (1), (2) with the cone diameter and the pyramid base increasing at the height of the cone or pyramid $0.5 \mu \mathrm{m}$. The effective photocarrier lifetime changes slightly at $0.05 \mu \mathrm{m}$, then abruptly drops at $0.2 \mu \mathrm{m}$ and then gradually saturates at $0.5 \mu \mathrm{m}$ (curves 1, 2 from Figure $a$ ). Saturation is not observed in Figure $b$, it will come at higher heights of cones and pyramids.

Experimental data for cones in Figure (signs) are in good correlation with calculations of the effective lifetime of minority carriers. One can obtain the same lifetime for pyramids at 7-8 times lower the base square and 1.4 times lower heights in comparison with cones.

\section{Conclusion}

The numerical calculation shows that the n-type polished plate of single silicon crystal and black silicon have high volume lifetimes and the minority carrier lifetime at the surface, indicating a high purity of material volume and surface. However, the surface lifetime is lower than the volume lifetime of minority carriers. So, the measured 
experimentally effective lifetime of minority carriers is determined by the surface lifetime.

The minority carrier lifetime in the black silicon wafer is comparable with the volume one. The surface lifetime reduced three times faster in the $n$-type polished and black silicon due to the high volume and surface lifetimes. Experimental lifetime of minority carriers for cones is in good correlation with calculations of the effective lifetime inherent to photocarriers. One can obtain the same lifetime for pyramids at lower sizes in comparison with that of cones.

\section{References}

1. Savin H., Repo P., Gastrow G., Ortega P., Calle E., Garín M., Alcubilla R. Black silicon solar cells with interdigitated back-contacts achieve $22.1 \%$ efficiency. Nature Nanotechnology. 2015. 10, No.7. P. 624-628.

2. Bett A.J., Eisenlohr J., Höhn O., Repo P., Savin H., Bläsi B., Goldschmidt J.C. Wave optical simulation of the light trapping properties of black silicon surface textures. Opt. Express. 2016. 24, No. 6. P. A434-A445.

3. Karachevtseva L.A., Lytvynenko O.O., Konin K.P., Parshyn K.A., Sapelnikova O.Yu., Stronska O.J. Electro-optical effects in 2D macroporous silicon structures with nanocoatings. Semiconductor Physics, Quantum Electronics \& Optoelectronics. 2015. 8, No. 4. P. 377-384.

4. Karachevtseva L.A., Kartel M.T., Lytvynenko O.O., Onyshchenko V.F., Stronska O.J. Polymernanoparticle coatings on macroporous silicon matrix. Adv. Mater. Lett. 2017. 8, No. 4. P. 336341.

5. Oh J., Yuan H.-C. \& Branz H.M. An 18.2\%efficient black-silicon solar cell achieved through control of carrier recombination in nanostructures. Nature Nanotechnology. 2012. 7, No. 11. P. 743 748.

6. Koynov S., Brandt M.S \& Stutzmann M. Black multi-crystalline silicon solar cells. phys. status solidi RRL. 2007. 1, No. 2. P. R53-R55.

7. Garnett E., Yang P. Light trapping in silicon nanowire solar cells. Nano Lett. 2010. 10, No. 3. P. 1082-1087.

8. Huang Z., Carey J.E., Liu M., Guo X., Mazur E., Campbell J.C. Microstructured silicon photodetector. Appl. Phys. Lett. 2006. 89, No. 3. P. 033506-033508.

9. Juntunen M.A., Heinonen J., Vähänissi V., Repo P., Valluru D., Savin H. Near-unity quantum efficiency of broadband black silicon photodiodes with an induced junction. Nature Photonics. 2016. 10, No. 12. P. 777-781.

10. Otto M., Algasinger M., Branz H., Geseman B. et al. Black silicon photovoltaics. Adv. Opt. Mater. 2015. 3, No. 2. P. 147-164.

11. Zhong S.H., Liu B.W., Xia Y., Liu J.H., Liu J., Shen Z.N., Xu Z., Li C.B. Influence of the texturing structure on the properties of black silicon solar cell. Sol. Energ. Mat. Sol. C. 2013. 108. P. 200-204.

12. Repo P., Haarahiltunen A., Sainiemi L., Yli-Koski M., Talvitie H. et al. Effective passivation of black silicon surfaces by atomic layer deposition. IEEE $J$. Photovolt. 2013. 3, No. 1. P. 90-94.

13. Otto M., Kroll M., Käsebier T., Salzer R., Tünnermann A., Wehrspohn R.B. Extremely low surface recombination velocities in black silicon passivated by atomic layer deposition. Appl. Phys. Lett. 2012. 100, No. 19. P. 191603-91606.

14. Gesemann B., Wehrspohn R., Hackner A. and Müller G. Large-scale fabrication of ordered silicon nanotip arrays used for gas ionization in ion mobility spectrometers. IEEE Trans. Nanotechnol. 2011. 10, No. 1. P. 50-52.

15. Hoyer P., Theuer M., Beigang R. and Kley E.-B. Terahertz emission from black silicon. Appl. Phys. Lett. 2008. 93, No. 9. P. 091106-1-091106-3.

16. Ernst M., Brendel R. Modeling effective carrier lifetimes of passivated macroporous silicon layers. Solar Energy Materials and Solar Cells. 2011. 95, No. 4. P. 1197-1202.

17. Onyshchenko V.F. Distribution of non-equilibrium charge carriers in macroporous silicon structure under conditions of their homogeneous generation over the simple bulk. Optoelectronics and Semiconductor Technique. 2015. 50. P. 125-131 (in Ukrainian).

18. Plakhotnyuk M., Schmidt D.R., Stenbæk S.M., Malureanu R., Stamate E., Hansen O. Lifetime of nano-structured black silicon for photovoltaic applications. Proc. 32-nd Europ. Photovolt. Solar Energy Conf. and Exhibition. IEEE. 2016. P. 764 767. 